\title{
Valutazione degli eventi cardio- e cerebrovascolari a lungo termine in pazienti con iperaldosteronismo primario
}

\author{
Emanuela Arvat
}

Pubblicato online: 11 marzo 2014

(C) Springer International Publishing AG 2014

Commento a:

Long-term cardio- and cerebrovascular events in patients with primary aldosteronism.

P. Mulatero, S. Monticone, C. Bertello, A. Viola, D. Tizzani, A. Iannaccone, V. Crudo, J. Burrello, A. Milan, F. Rabbia and F. Veglio.

J Clin Endocrinol Metab 98:4826-4833, 2013

L'aldosterone induce importanti danni diretti a livello cardiovascolare $(\mathrm{CV})$, indipendenti dall'entità dei livelli pressori. Numerosi studi hanno evidenziato un aumento della prevalenza di complicanze e di eventi $\mathrm{CV}$ in pazienti affetti da iperaldosteronismo primario (IA) rispetto a quelli con ipertensione essenziale (IE) alla diagnosi, mentre non è del tutto chiaro l'andamento degli eventi CV durante il follow-up a lungo termine (mediana 12 aa) di questi pazienti.

Questo studio ha valutato 270 pazienti affetti da IA (161 maschi e 109 femmine, età $44 \pm 8,5$ aa) suddivisi in due gruppi in base alla tipologia di IA: 57 con adenoma surrenalico aldosterone-secernente (APA) e 213 con iperplasia surrenalica bilaterale $(\mathrm{BAH})$, diagnosticati mediante imaging e cateterismo delle vene surrenaliche. I pazienti sono stati accoppiati nel rapporto 1:3 con 810 controlli affetti da IE, sovrapponibili per sesso, età, stile di vita, BMI, valori pressori, durata di malattia, quadro metabolico.

È stata confrontata la percentuale di eventi CV sia alla diagnosi sia durante il follow-up tra i pazienti affetti da IA e quelli con IE e, nell'ambito dei pazienti con IA, tra quelli con APA o con BAH.

\section{E. Arvat $(\varangle)$}

Divisione di Endocrinologia Oncologica, Dipartimento di Scienze

Mediche, Università di Torino, Torino, Italia

e-mail: emanuela.arvat@unito.it
Nel corso dell'intera osservazione, i pazienti con IA hanno dimostrato un' aumentata insorgenza di eventi $(22,6 \%$ vs $12,7 \%$ ); alla diagnosi, i pazienti con IA hanno dimostrato un'aumentata incidenza di eventi nella loro storia clinica $(14,1 \%$ vs $8,4 \%, p<0,007)$ e tale differenza è stata riscontrata anche nel corso del follow-up (eventi in IA vs IE: $8,5 \%$ vs $4,3 \%, p<0,008)$. La tipologia degli eventi CV maggiormente riscontrati nei pazienti con IA includeva l'insorgenza di infarto miocardico, stroke, aritmie atriali e ventricolari, insufficienza cardiaca, nonostante un miglior profilo lipidico osservato in questi pazienti. Inoltre, durante il follow-up, un maggior numero di pazienti con IA ha dimostrato l'insorgenza di diabete mellito tipo 2. Il confronto fra APA a BAH ha dimostrato che i pazienti con APA presentavano un maggior numero di pregressi eventi $\mathrm{CV}$ alla diagnosi ma non durante il follow-up, suggerendo che l'intervento di surrenectomia e la normalizzazione di livelli di aldosterone negli APA fosse cruciale nel ridurre nel tempo il rischio $\mathrm{CV}$. Peraltro, in entrambi tale rischio risultava superiore a quello riscontrato nei pazienti con IE.

Nel complesso, tali risultati confermano e rinforzano l'ipotesi del danno diretto da parte dell'aldosterone, indipendentemente dai livelli pressori, sulla funzione cardiovascolare, che non è completamente reversibile dopo la normalizzazione dei livelli ormonali ottenuta con la surrenectomia. I risultati dello studio dimostrano, inoltre, un effetto negativo delle elevate concentrazioni ormonali sul metabolismo glicidico, che si ipotizza essere mediato da un'attivazione dei meccanismi infiammatori locali, un aumento dello stress ossidativo cellulare, una riduzione della sensibilità all'insulina, sebbene non si possa escludere un'azione indiretta mediata dalla concomitante attivazione della secrezione glucocorticoide frequentemente osservata nei pazienti iperaldosteronemici. 